\title{
Ultrasound combined with nanobubbles promotes systemic anticancer immunity and augments anti-PD1 efficacy
}

\author{
Jianjun Hu (D) , ${ }^{1}$ Jiangyi He, ${ }^{1}$ Yunlong Wang, ${ }^{1}$ Yang Zhao, ${ }^{1}$ Kejing Fang, ${ }^{2}$ Yan Dong, \\ Yanrong Chen, ${ }^{1}$ Yue Zhang, ${ }^{1}$ Chi Zhang, ${ }^{1}$ Hongwei Wang, ${ }^{1}$ Jun Tan, ${ }^{1}$ Junyi Wang, ${ }^{1}$ \\ Ruiyang Zi, ${ }^{1}$ Chengxiang Liu, ${ }^{1}$ Houjie Liang, ${ }^{1}$ Yanli Guo, ${ }^{2}$ Juanjuan $\mathrm{Ou}^{1}$
}

To cite: Hu J, He J, Wang Y, et al. Ultrasound combined with nanobubbles promotes systemic anticancer immunity and augments anti-PD1 efficacy. Journal for ImmunoTherapy of Cancer 2022;10:e003408. doi:10.1136/jitc-2021-003408

- Additional supplemental material is published online only. To view, please visit the journal online (http://dx.doi.org/10. 1136/jitc-2021-003408).

$\mathrm{JHu}, \mathrm{JHe}, \mathrm{YW}$ and $\mathrm{YZ}$ are joint first authors.

Accepted 28 January 2022

Check for updates

(C) Author(s) (or their employer(s)) 2022. Re-use permitted under CC BY-NC. No commercial re-use. See rights and permissions. Published by BMJ.

${ }^{1}$ Department of Oncology, Army Medical University, Chongqing, China

2Department of Ultrasound, Army Medical University, Chongqing, China

\section{Correspondence to}

Professor Houjie Liang; lianghoujie@sina.com

Professor Yanli Guo; guoyanli71@aliyun.com

Professor Juanjuan 0u; 13957751@qq.com

\section{ABSTRACT}

Background The poor immunogenicity of solid tumors limits the efficacy ofanti-programmed cell death protein 1 (anti-PD1)-based immune checkpoint blockade (ICB); thus, less than $30 \%$ of patients with cancer exhibit a response. Currently, there is still a lack of effective strategies for improving tumor immunogenicity.

Methods The antitumor effect of ultrasound-stimulated nanobubbles (USNBs) alone and in combination with an anti-PD1 antibody was evaluated in RM1 (prostate cancer), MC38 (colon cancer) and B16 (melanoma) xenograft mouse models. The phenotypes of antigen-presenting cells and CD8+ T cells were evaluated by flow cytometry. Damage-associated molecular pattern (DAMP) release, antigen release and tumor cell necrosis were assessed via western blot, flow cytometry, transmission electron microscopy and confocal microscopy.

Results USNB promoted the infiltration and antitumor activity of CD8+ T cells. The combination of USNB and anti-PD1 blockade improved systemic antitumor immunity and resulted in an abscopal effect and long-term immune memory protection after complete tumor remission. Mechanistically, tumor-targeting USNB induced tumor cell necrosis through an ultrasound-mediated cavitation effect, which significantly increased DAMP release and tumor antigen presentation, consequently sensitizing tumors to ICB treatment

Conclusion The administration of USNB increased tumor immunogenicity by remodeling the tumor-immune microenvironment, providing a promising strategy for sensitizing poorly immunogenic solid tumors to immunotherapy in the clinic.

\section{INTRODUCTION}

Immune checkpoint blockade (ICB) represents a breakthrough in cancer therapy and acts by reinvigorating the cytotoxic potential of the immune system. ${ }^{1-3}$ However, the clinical efficacy of ICB remains very limited, leaving most patients unable to benefit from such treatment. ${ }^{4-6}$ Increasing evidence has revealed primary resistance mechanisms which include insufficient tumor immunogenicity, immunosuppression and inadequate T-cell activation. It has been reported that a sufficient pre-existing population of antitumor CD8+ $\mathrm{T}$ cells within the tumor microenvironment (TME) favors tumor susceptibility to PD1-based ICB therapy. ${ }^{78}$ Among patients with melanoma who undergo anti-PD1 therapy, the presence of sufficient T-cell numbers in biopsy specimens prior to treatment is associated with a better response to therapy, ${ }^{9}$ and the CD8+ T-cell density at the tumor margin was predictive of a good response,${ }^{10}$ suggesting a positive correlation between intratumoral T-cell infiltration and sensitivity to ICB therapy. Previous studies have demonstrated that the lack of immunogenicity of solid tumors is an obstacle to their sensitivity to ICB therapy, but how to improve immunogenicity remains largely unknown.

The recent classification of 'hot' and 'cold' tumors has been increasingly advocated as an aid to the clinical application of ICB. ${ }^{11-13}$ Cold tumors, such as prostate cancer and colon cancer, do not respond to single-agent ICB therapies because of their low immunogenicity. ${ }^{13-18}$ Combination therapy has been used in the clinic to turn cold tumors into hot tumors in order to increase the efficacy of immunotherapy in recent years; such combination therapies include chemotherapy or radiation therapy combined with immunotherapy. ${ }^{19} 20$ Chemotherapy and radiation can mainly lead to tumor cell apoptosis by disrupting cell division or destroying mitochondria. However, apoptosis is a type of physiological cell death associated with poor immunogenicity. ${ }^{21-23}$ It is of vital importance to identify new combination therapies to improve the benefit of ICB.

Lipid microbubbles (MBs) encapsulating inert gas were originally used as contrast agents for low-power ultrasound (US) imaging. ${ }^{24}$ It has been reported that US-stimulated $\mathrm{MB}$ cavitation can promote tumor perfusion, thereby increasing CD8+ T-cell 


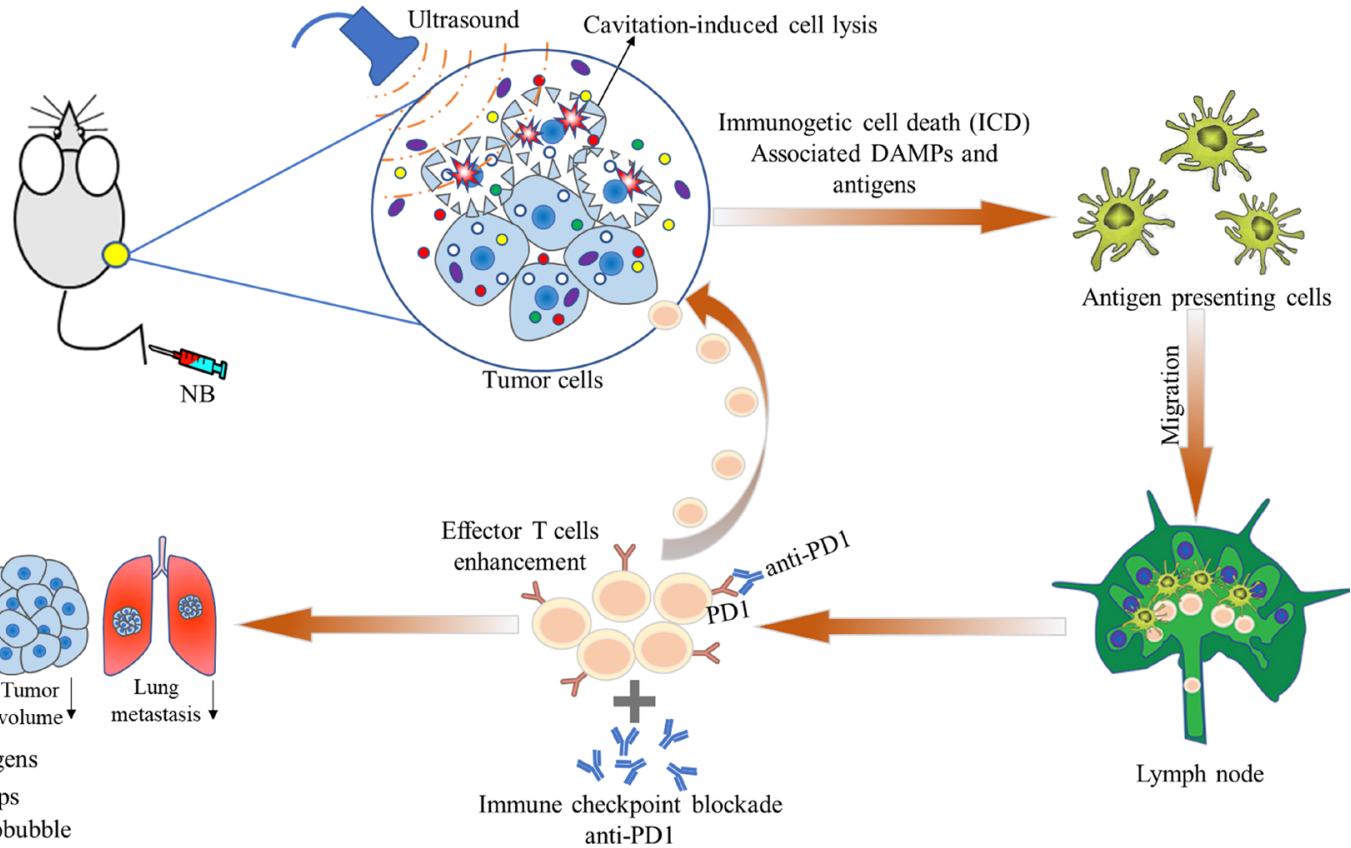

Figure 1 Schematic illustration showing the effects of ultrasound-stimulated NB on a mouse tumor model. NBs are injected into mice via the tail vein; the NB can then circulate into the tumor microenvironment and taken up by tumor cells. After US is applied, the destruction of the NB can cause tumor cell lysis and the release of immunogenic substances from cells, further activating innate and adaptive immune cells and eventually activating CD8+ T cells; this leads to systemic antitumor immunity and enhances the therapeutic effect of anti-PD1 treatment. DAMP, damage-associated molecular pattern; NB, nanobubble.

infiltration and enhancing the efficacy of ICB in an MC38 tumor model. ${ }^{25}{ }^{26}$ Since MB can only minimally enter the TME because the gaps between the endothelial cells in tumor blood vessels are smaller than $2 \mu \mathrm{m}$, their application to tumor immunogenicity remains limited. ${ }^{27} 28$ Compared with $\mathrm{MB}$, nanobubbles (NBs) can easily pass through the blood vessel endothelium and accumulate in the tumor mass. ${ }^{29}$ In addition, we have demonstrated that NBs destroyed by sufficient ultrasonic power show minor thermal effects. ${ }^{30} 31$ Previous studies have proven that NB carrying Ce6 (a sonosensitizer) suppress cancer cells through sonosensitizer-induced reactive oxygen species (ROS) generation, thereby achieving a synergistic effect with PD1 blockade ${ }^{21}$;2; however, the impact of ultrasoundstimulated nanobubbles (USNBs) on the tumor-immune microenvironment and their implication in tumor immunogenicity remain largely elusive.

Here, we revealed for the first time that USNB can effectively transform cold tumors into hot tumors, thereby exerting a synergistic effect with anti-PD1 treatment on 'cold tumors'. Importantly, we demonstrated that USNB significantly increase the release of antigens and damageassociated molecular pattedamage-associated molecular patternrns (DAMPs) to activate the inflammatory response in the TME and draining lymph nodes (dLNs), resulting in enhanced systemic antitumor immunity and immune memory and causing long-lasting inhibition of tumor growth and recurrence (figure 1). These findings strongly suggest a promising role of USNB in improving the efficacy of anti-PD1 therapy in cold tumors in the clinic.

\section{METHODS}

\section{Cell lines and animals}

The murine prostate cancer cell line RM1, an ovalbumin (OVA)-expressing RM1 cell line (RM1-OVA), the colon carcinoma cell line MC38, an ovalbuminexpressing MC38 cell line (MC38-OVA) and the melanoma cell line B16 were maintained in our laboratory. RM1, RM1-OVA, MC38, MC38-OVA and B16 cells were maintained in Dulbecco's Modified Eagle Medium (DMEM) (HyClone, Cytiva) supplemented with 10\% fetal bovine serum (HyClone, Cytiva). All the cell lines were cultured in medium supplemented with $100 \mu \mathrm{g} / \mathrm{mL}$ penicillin and $50 \mu \mathrm{g} / \mathrm{mL}$ streptomycin in a humidified $5 \% \mathrm{CO}_{2}$ incubator at $37^{\circ} \mathrm{C}$ and passaged using standard cell culture techniques. Female $\mathrm{C} 57 \mathrm{BL} / 6$ and nude mice were purchased from Hua Fukang (Beijing, China) and subsequently housed at the Animal Center of Southwest Hospital (Chongqing, China) under specific pathogen free (SPF) conditions.

\section{Preparation of lipid NBs}

The lipid components 1,2-dipalmitoyl-sn-glycero-3-ph osphocholine (Corden Pharma, LIestal, Switzerland), 1,2-dipalmitoyl-sn-glycero-3-phosphatidic acid (Corden Pharma), 1,2-dipalmitoyl-sn-glycero-3-phosphoethanol amine (Corden Pharma), 1,2-dipalmitoyl-sn-glycero-3-p hosphoglycerol (Corden Pharma) and DSPE-PEG2000$\mathrm{COOH}$ (Corden Pharma) were dissolved in a hydration solution of glycerine and phosphate-buffered saline (PBS) at a ratio of 1:9 (volume:volume) and incubated overnight in a horizontal shaker at $37^{\circ} \mathrm{C}$. The solution was 
transferred to a cillin bottle; the air inside was replaced by perfluoropropane (C3F8; Research Institute of Physical and Chemical Engineering of Nuclear Industry, Tianjin, China), and the solution was vortexed on an ST Amalgam Capsule Blender for $90 \mathrm{~s}$. The vibrated solution was centrifuged at $300 \mathrm{rpm}$ for $5 \mathrm{~min}$ to remove MBs from the supernatant. The particle size and zeta potential of the NBs were measured on a Malvern Zetasizer nano ZS90 detector.

\section{Cellular uptake imaging}

RM1 cells $\left(1 \times 10^{5}\right)$ and MC38 cells $\left(1 \times 10^{5}\right)$ were seeded in a glass-bottomed $35 \mathrm{~mm}$ cell culture dish and incubated for 24 hours. The cells were washed with PBS and incubated with 3,3'-dioctadecyloxacarbocyanine perchlorate (DiO)-labeled NB in serum-free RPMI medium for 5, 15 or $30 \mathrm{~min}$. The cells were then washed twice with PBS and stained with 4',6-diamidino-2-phenylidole (DAPI) for 5 min. Then, the cells were washed twice with PBS and promptly analyzed using a confocal laser scanning microscope (ZEISS LSM880) to visualize DAPI staining (blue color, $\left.\lambda_{\mathrm{Ex}}=405 \mathrm{~nm}, \lambda_{\mathrm{Em}}=450-500 \mathrm{~nm}\right)$ and DiO-labeled NB (red color, $\lambda_{\mathrm{Ex}}=488 \mathrm{~nm}, \lambda_{\mathrm{Em}}=500-560 \mathrm{~nm}$ ).

\section{Annexin V/7-Amino-Actinomycin D (7AAD), Caspase3, R0S assay}

RM1 cells and MC38 cells $\left(2 \times 10^{5}\right)$ were seeded in six-well plates and incubated for 24 hours. Then, the cells were divided into four groups: the untreated group, US-treated group (the US was generated by a WED-100 ultrasonic therapy machine, and the machine parameters were set as follows: effective sound intensity: $1 \mathrm{~W} / \mathrm{cm}^{2}$, irradiation time: $5 \mathrm{~s}$, working mode: pulse mode, pulse duration: $6 \mathrm{~ms}$, pulse repetition cycle: $10 \mathrm{~ms}$, sound frequency: 1 $\mathrm{MHz}$, beam type: collimation, and effective radiation area of the probe: $2.0 \mathrm{~cm}^{2}$. To minimize the influence of the standing wave, the ultrasonic probe was in full contact with the active part through a coupling agent and moved around the action site during treatment), NB-incubated group (5 min incubation) and USNB group (NB incubated for $5 \mathrm{~min}$ and then treated with US; the ultrasonic settings were the same as those used in the US-treated group). For annexin $\mathrm{V} / 7 \mathrm{AAD}$ and caspase 3 staining, the cells were harvested at 0 or 4 hours after treatment and stained with annexin V (88-8102-72, eBioscience), 7AAD (00-6993-50, eBioscience) and caspase3 (550914, BD Biosciences) according to the manufacturers' instructions. For ROS staining, the cells were harvested 4 hours after treatment and stained for ROS (C10422, Thermo Fisher) according to the manufacturer's instructions. The stained cells were evaluated by flow cytometry (NovoCyte, ACEA), and the flow cytometry data were analyzed with FlowJo software (Tree Star).

\section{Carboxyfluorescein succinimidyl ester (CFSE) assay}

CFSE (C34554, Invitrogen) was added to RM1 cells $\left(2 \times 10^{7}\right)$ and MC38 $\left(2 \times 10^{7}\right)$ cells suspended in $10 \mathrm{~mL}$ medium at a final concentration of $5 \mu \mathrm{M}$. After a $20 \mathrm{~min}$ incubation in humidified air with $5 \% \mathrm{CO}_{2}$ at $37^{\circ} \mathrm{C}, 40 \mathrm{~mL}$ fluorescence activated cell sorting (FACS) buffer was added to remove the unbound dye, and then, the cells were divided equally into four groups as mentioned previously. After treatment, the cells were cultured for 24 hours. Then, the cells were assessed by flow cytometry (NovoCyte, ACEA).

\section{ATP assay}

Extracellular ATP was quantified using a luciferinluciferase-based ATP assay kit (S0026; Beyotime Institute of Biotechnology, China). RM1 cells $\left(1 \times 10^{6}\right)$ and MC38 cells $\left(1 \times 10^{6}\right)$ were suspended in $200 \mu \mathrm{L}$ of PBS. After the previously described treatment, the cells were centrifuged; $20 \mu \mathrm{L}$ of the supernatant was added to the luciferin substrate and luciferase enzyme mixture; and the bioluminescence intensity was immediately read with a fluorescence microplate reader (Varioskan Flash, Thermo Scientific).

\section{Immunofluorescence}

MC38 cells were divided into four groups and treated as mentioned previously. After treatment, the cells were collected in $1.5 \mathrm{~mL}$ microcentrifuge tubes, stained with MitoTracker (M7514, Thermo Fisher) at room temperature for $20 \mathrm{~min}$, washed with PBS three times, resuspended in $200 \mu \mathrm{L}$ PBS, stained with 7AAD at room temperature for $5 \mathrm{~min}$ and placed on a glass-bottomed $35 \mathrm{~mm}$ cell culture dish. The cells were analyzed using a confocal laser scanning microscope (ZEISS LSM880).

\section{Transmission electron microscopy (TEM)}

$\mathrm{NB}$ and US-stimulated NB were prepared as described earlier. A lacey copper grid coated with carbon film was dipped into the samples, removed and then stained with phosphotungstic acid. The four groups of RM1 cells and MC38 cells were treated as described previously. Immediately after treatment, the cells were collected and fixed in $2.5 \%$ glutaraldehyde, postfixed in $1 \%$ osmium tetroxide and embedded in resin. The samples were poststained with saturated uranium dissolved in $70 \%$ acetone. Ultrathin sections were generated and then stained with lead citrate. All the specimens were mounted on a holder matched with the TEM column. The specimen and holder tip were imaged on a JEM-1400 Plus electron microscope operated at $100 \mathrm{kV}$ at a magnification in the range of $\times 15000-20000$ to assess the NB size and distribution and at a magnification in the range of $\times 2500-50000$ to assess cell and organelle structure. All the images were captured with a Gatan ORIUS SC 200D CCD camera.

\section{Western blotting}

Western blotting was performed with primary antibodies specific for the following molecules: HMGB1 (ab18265, Abcam), calreticulin (ab92516, Abcam), HSPA2 (bs-18080R, Bioss) and OVA (10 R-1905, Fitzgerald). RM1OVA $\left(1 \times 10^{6}\right)$ and MC38-OVA cells $\left(1 \times 10^{6}\right)$ resuspended in $1 \mathrm{~mL}$ medium were treated as described previously. After treatment, the cell suspensions were centrifuged at $2000 \mathrm{~g}$ at $4^{\circ} \mathrm{C}$ for $5 \mathrm{~min}$ to obtain the supernatants. The 
supernatants were further concentrated to $40 \mu \mathrm{L}$ with a centrifugal filter (UFC803024; Amicon Ultra4, Millipore, Merck) and subjected to western blotting.

\section{Tumor inoculation and therapy}

RM1 cells $\left(1 \times 10^{6}\right)$, MC38 cells $\left(0.5 \times 10^{6}\right)$ or B16 cells $\left(1 \times 10^{6}\right)$ in $100 \mu \mathrm{L}$ PBS were subcutaneously injected into the right legs of 6-week-old mice. When the tumors reached $50 \mathrm{~mm}^{3}$ (approximately 8 days after RM1 and B16 tumor cell inoculation and 3 days after MC38 tumor cell inoculation), the mice were divided into four groups: the untreated, USNB, anti-PD1 and USNB+anti-PD1 groups. NB $(100 \mu \mathrm{L})$ were intravenously administered on the day of treatment, and US was applied to the tumors $5 \mathrm{~min}$ after NB injection. The US was generated by a WED-100 ultrasonic therapy machine, and the machine parameters were set as follows: effective sound intensity: $1 \mathrm{~W} /$ $\mathrm{cm}^{2}$, irradiation time: $30 \mathrm{~s}$, working mode: pulse mode, pulse duration: $6 \mathrm{~ms}$, pulse repetition cycle: $10 \mathrm{~ms}$, sound frequency: $1.0 \mathrm{MHz}$, beam type: collimation and effective radiation area of the probe: $2.0 \mathrm{~cm}^{2}$. To minimize the influence of the standing wave, the ultrasonic probe was in full contact with the active part through a coupling agent and moved around the action site during treatment. Furthermore, anti-PD1 (200 $\mu \mathrm{g}$ in PBS) was started at the same time and was intraperitoneally injected into the mice once every 3 days during the treatment period for a total of four doses. The tumor volume was calculated as $\mathrm{V}_{\mathrm{T}}=0.5 \times \mathrm{L}$ (largest diameter) $\times \mathrm{W}$ (smallest diameter). ${ }^{2}$ The endpoint of the study was when the tumor reached $20 \mathrm{~mm}$ in diameter or $2000 \mathrm{~mm}^{3}$ in volume.

\section{RNA-sequencing (RNAseq)}

RM1 cells $\left(1 \times 10^{6}\right)$ in $100 \mu \mathrm{L}$ PBS were subcutaneously injected into the right legs of 6-week-old C57BL/ 6 mice, and the mice were treated with USNB, anti-PD1 and USNB+anti-PD1. At day 18, we sacrificed the mice and performed transcriptomics analysis using separated tumor mass. Total tumor's RNA was extracted and analyzed by Shanghai Jiayin Biotechnology Co. A total amount of $3 \mu \mathrm{g}$ RNA per sample was used as input material for the RNA sample preparations. Sequencing libraries were generated using the NEBNext Ultra RNA Library Prep Kit for Illumina (NEB, USA) following the manufacturer's recommendations. Gene ontology (GO) analysis was performed to elucidate the biological implications of the unique genes in the significant differentially expressed gene profiles in this experiment. We downloaded the GO annotations from NCBI (http:/ /www.ncbi.nlm.nih.gov/), UniProt (http://www.uniprot.org/) and Gene Ontology (http://www.geneontology.org/). Fisher's exact test was used to identify the significant GO categories, and false discovery rate (FDR) was used to correct the $\mathrm{p}$ values.

\section{Antitumor efficacy against abscopal tumors}

RM1 cells $\left(1.2 \times 10^{6}\right)$ or MC38 cells $\left(0.7 \times 10^{6}\right)$ were subcutaneously injected into the right legs of 6 -week-old mice, and 3 days later, $1 \times 10^{6} \mathrm{RM} 1$ cells and $0.5 \times 10^{6} \mathrm{MC} 38$ cells were subcutaneously injected into the opposite legs of the mice, respectively. To observe lung metastasis, $0.3 \times 10^{6}$ MC38 cells were intravenously injected into the mice via the tail vein 3 days after cell inoculation into the right legs. When the tumors on the right legs reached $50 \mathrm{~mm}^{3}$, the mice were divided into four groups and treated as described earlier. US was applied to the tumor on the right side.

\section{Histology}

Tumors were collected from the mice after the endpoint of the study and fixed in $4 \%$ paraformaldehyde. The fixed tumor tissues were embedded in paraffin, sectioned to a thickness of approximately $5 \mu \mathrm{m}$ and stained with H\&E. The sections were examined with a ZEISS AxioScan Z1 microscope, and the images were analyzed with ZEN software.

\section{Flow cytometry}

The following Abs were used: anti-CTLA-4 (HMCD15201, Thermo Fisher), anti-CD39 (143804, BioLegend), anti-CD8 (553031, BioLegend), anti-CD73 (127220, BioLegend), anti-Tbet (644810, BioLegend), anti-CD44 (103030, BioLegend), anti-KLRG1 (138414, BioLegend), anti-CD11b (101230, BioLegend), anti-CXCR5 (551961, BD Biosciences), anti-CD25 (564571, BD Biosciences), anti-CD4 (553052, BD Biosciences), anti-CD107a/b (553793/558758, BD Biosciences), anti-B220 (561102, BD Biosciences), anti-PD1 (11-9985-81, eBioscience), antiFoxp3 (50-5773-82, eBioscience), anti-CD11c (17-011482, eBioscience), anti-CD45 (12-0451-82, eBioscience), anti-CD49b (25-5971-82, eBioscience) and anti-TCF-1 (2206S, Cell Signaling Technology). Fixable Viability Dye (65-0865-14, eBioscience)-stained cells were excluded from analysis. Tumor-bearing mice were sacrificed; the tumors were sliced and digested with type I collagenase (A004194-0001, Sangon Biotech) for 1 hour at $37^{\circ} \mathrm{C}$; and the spleens and dLNs were ground to generate singlecell suspensions. The single-cell suspensions were filtered through $70 \mu \mathrm{m}$ strainers (352350, BD Biosciences) and stained as described. The stained cells were evaluated by BD FACS Canto II flow cytometry, and the flow cytometry data were analyzed with FlowJo software (Tree Star).

\section{Depletions}

Depletions of cellular subsets in vivo were carried out using antibodies against CD8 (100764, BioLegend), CD4 (100458, BioLegend) and NK1.1 (108759, BioLegend).

\section{Ex vivo coculture assay}

A total of $1 \times 10^{7}$ splenocytes from RM1 or MC38 tumorbearing C57BL/6 mice were isolated. These splenocytes were cocultured with $200 \mu \mathrm{L}$ supernatants from USNB, US, NB and untreated MC38 tumor cells in a total volume of $1 \mathrm{~mL}$ medium supplemented with $20 \mathrm{ng} / \mathrm{mL}$ interleukin-2 (RP-8605, eBioscience). The function of CD8+ T cells was assessed by flow cytometry 3 days later. 


\section{Statistical analysis}

An unpaired two-tailed non-parametric t-test with a 95\% CI was used to assess between-group differences. All the statistical calculations were performed using GraphPad Prism (GraphPad Software). The results are expressed as the mean \pm SEM.

\section{RESULTS \\ USNB synergistically improved the efficacy of anti-PD1 therapy}

Competent NBs with a uniform nanoscale grain diameter were synthesized as described in the experimental section. The morphology of the NBs before and after US treatment was visualized by TEM (online supplemental figure S1A). The general grain diameters ranged from 200 to $800 \mathrm{~nm}$ and were predominantly $(40 \%) 300-400 \mathrm{~nm}$ (online supplemental figure S1B), and the zeta potential of the NBs was $-1.42 \pm 0.549$. To evaluate whether USNB treatment can inhibit tumor growth and exerts a synergistic effect with PD1 blockade, we established a model of RM1 prostate cancer, which is considered a characteristic cold mouse tumor, in C57BL/6 mice and treated the mice with USNB, anti-PD1 or USNB+anti-PD1 (online supplemental figure S1C). USNB treatment slowed the growth of tumors; however, anti-PD1 treatment exerted a weak therapeutic effect when used alone. As we expected, the USNB and anti-PD1 combination treatment significantly inhibited tumor growth (figure 2A). We further evaluated USNB treatment in MC38 colon cancer and B16 melanoma cells. Anti-PD1 monotherapy did not efficiently delay tumor growth in the B16 tumor model but showed a moderately good effect in the MC38 tumor model. USNB treatment partially reduced the growth rate of both tumors, whereas the combination treatment obviously slowed the growth of MC38 (figure 2B) and B16 (figure 2C) tumors. The individual animal tumor growth curves of RM1, MC38 and B16 for each treatment condition are shown in online supplemental figure S1D-F, respectively. Tumor weights at the endpoint of the study showed the same trend (online supplemental figure S1GS1I). In addition, the combination treatment also significantly prolonged the survival rate of tumor-bearing mice (figure 2D-F). These results collectively confirmed that USNB treatment can delay tumor growth and enhance the therapeutic effect of PD1 blockade.

As the efficiency of the anti-PD1 antibody relies on the number and effector function of CD8+ T cells in the TME, we further investigated the presence of tumor-infiltrating CD44+CD8+ T cells in the RM1 and MC38 tumor models after the different treatments. Compared with that in the untreated group, the number of CD44+CD8+ T cells in RM1 tumors was increased in both the USNB and USNB+anti-PD1 groups but not in the anti-PD1 group (figure 2G). To determine whether CD44+CD8+ T-cell numbers were systemically increased, we further examined the number of $\mathrm{CD} 44+\mathrm{CD} 8+\mathrm{T}$ cells in the dLNs and spleen. In the USNB+anti-PD1 treatment group, the number of CD44+CD8+ T cells was apparently increased compared with that in the anti-PD1 and untreated groups (figure 2H,I), which indicates that the combined treatment leads to a systemic enhancement in T-cell immunity. Although there was an increased number of CD44+CD8+ $\mathrm{T}$ cells in the dLNs and spleens after anti-PD1 treatment, no significant change was observed in the tumors, revealing that single ICB treatment is inadequate in cold tumors. The data of the MC38 model are shown in online supplemental figure S2A and are generally consistent with the RM1 tumor model. Collectively, these results indicate that USNB treatment can promote the infiltration of CD8+ T cells and can strengthen systemic T-cell immunity, both of which provide a solid foundation for successful anti-PD1 treatment.

To investigate whether CD4+ $\mathrm{T}$ cells played a role in promoting the effect of USNB treatment, we further examined tumor-infiltrating CD4+ $\mathrm{T}$ cells and found that the proportions and numbers of total CD4+ T cells were comparable among the different treatment groups (figure 2J); similar results were observed for Th1 cells (figure 2K), which are crucial helper cells for CD8+ T-cell function, and immunosuppressive Treg cells (figure 2L). The corresponding data of the MC38 model are shown in online supplemental figure S2B-S2D. To further investigate whether $\mathrm{CD} 4+\mathrm{T}$ cells or $\mathrm{CD} 8+\mathrm{T}$ cells play the dominant role in USNB treatment effects, we applied CD4+ and CD8+ T cell-depleting antibodies in combination with USNB treatment and separated the mice into the USNB $+\alpha \mathrm{CD} 4, \mathrm{USNB}+\alpha \mathrm{CD} 8, \mathrm{USNB}+\alpha \mathrm{CD} 4+\alpha \mathrm{CD} 8$ and USNB groups. Because natural killer (NK) cells also protect against cancer, ${ }^{33}$ we added a USNB $+\alpha$ NK group in our experiment (online supplemental figure S2E). The mice in the USNB $+\alpha C D 4+\alpha \mathrm{CD} 8$ and USNB $+\alpha \mathrm{CD} 8$ groups exhibited the fastest tumor growth rates, and the mice in the USNB $+\alpha \mathrm{CD} 4$ and USNB $+\alpha N K$ groups exhibited a moderate tumor growth rate compared with those in the other groups (figure 2M). These results suggested that the effect of USNB relies mainly on CD8+ T cells.

\section{USNB endows CD8+ T cells with competence for immunotherapy}

As we confirmed that the number of tumor-infiltrating CD44+CD8+ T cells increased after USNB and combination treatment, we further investigated the effect function of infiltrating CD8+ T cells in the different treatment groups. USNB combined with PD1 blockade significantly increased the numbers of Granzyme B (GZMB)+CD8+ $\mathrm{T}$ cells, CD107+CD8+ $\mathrm{T}$ cells and interferon gamma $(\mathrm{IFN}-\gamma)+\mathrm{CD} 8+\mathrm{T}$ cells compared with USNB or PD1 blockade treatment alone (figure $3 \mathrm{~A}-\mathrm{C}$ ). The mean fluorescence intensities (MFIs) of GZMB, CD107, IFN- $\gamma$ and KLRG1 in CD44+CD8+ T cells were also increased after combination treatment (figure 3D). These results indicate that USNB treatment can enhance the effector function of tumor-infiltrating CD8+ T cells and further enhance the effect of PD1 blockade. Next, we examined the level of Tbet expression in CD44+CD8+ T cells. Tbet 

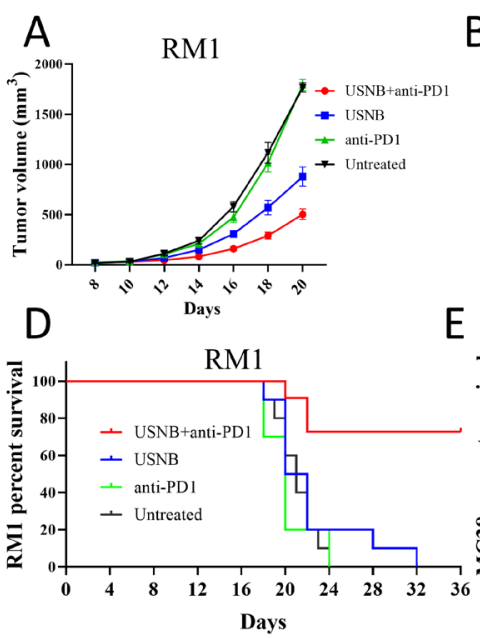

G

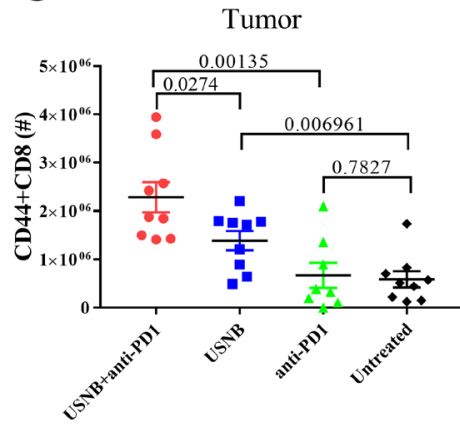

B

E

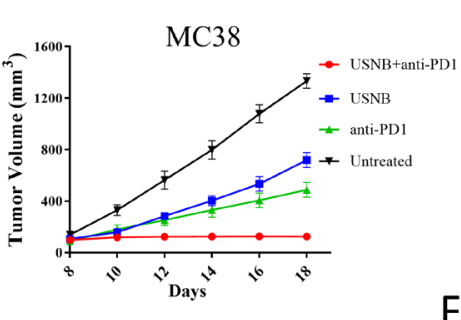

F
C
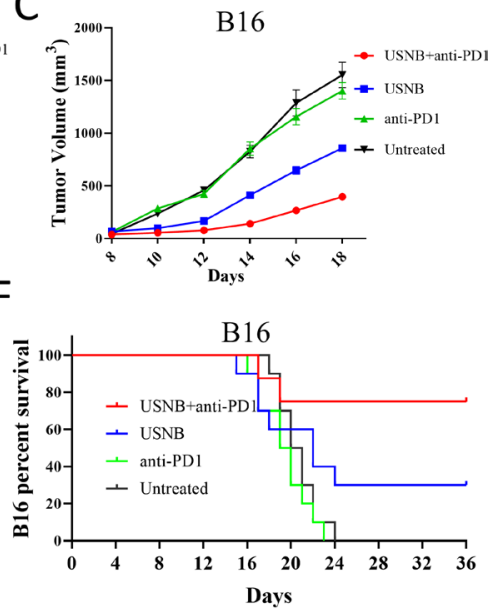

$\mathrm{H}$

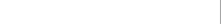

|
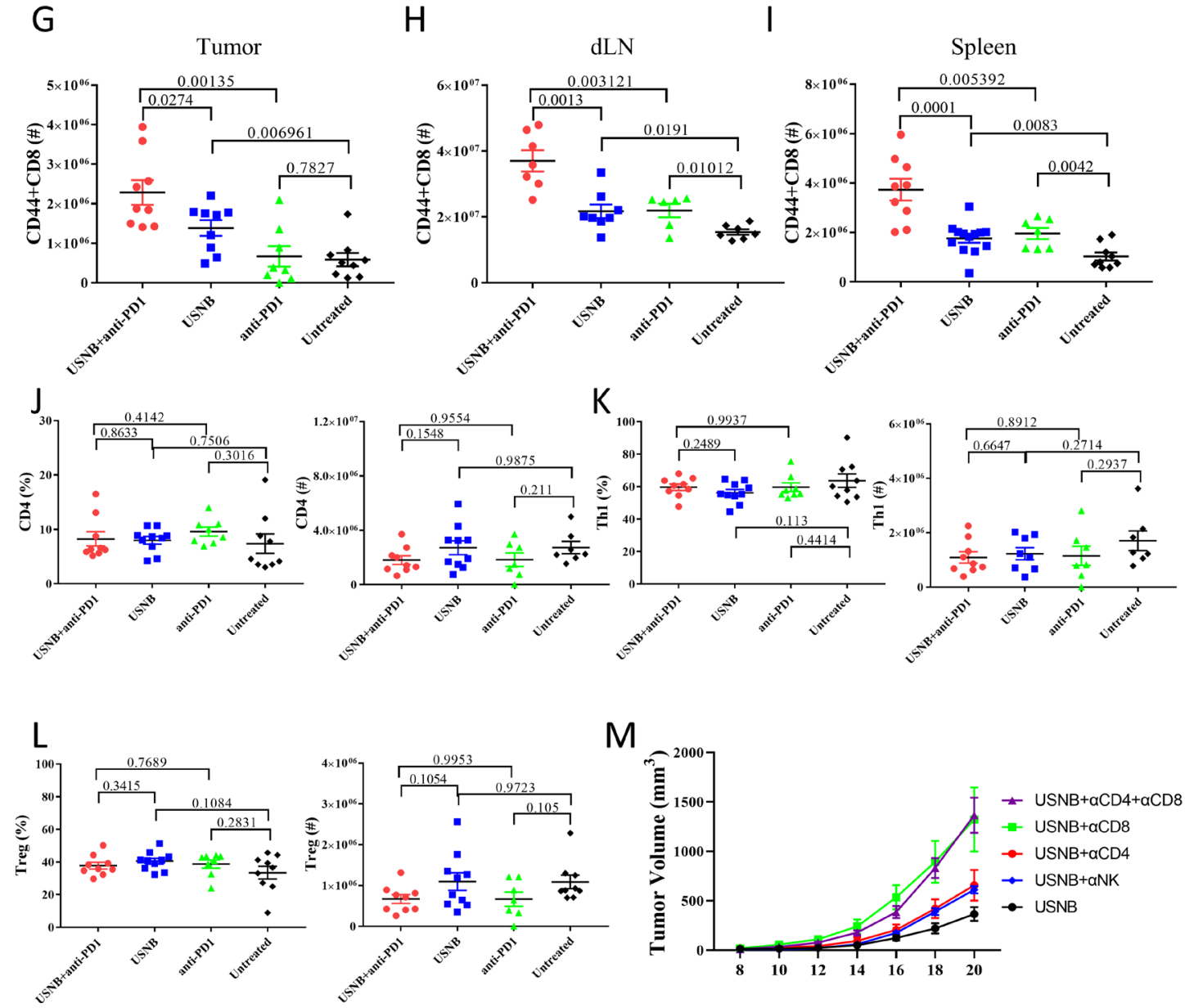

Figure 2 USNB enhances the therapeutic effect of PD1 blockade and promotes immune cell infiltration. Growth curve of C57BL/6 mice subcutaneously inoculated with $1 \times 10^{6} \mathrm{RM} 1$ cells $(\mathrm{A}), 0.5 \times 10^{6} \mathrm{MC} 38$ cells $(\mathrm{B})$ and $1 \times 10^{6} \mathrm{~B} 16 \mathrm{cells}(\mathrm{C})$. Survival curves of RM1 (D), MC38 (E) and B16 (F) tumor-bearing mice. Flow cytometry analysis of the number of CD44+CD8+ T cells in the tumors $(\mathrm{G})$, dLNs $(\mathrm{H})$ and spleens $(\mathrm{I})$. The percentage and absolute number of CD4+ T cells $(\mathrm{J})$, Th1 (K) and Treg $(\mathrm{L})$ cells in tumors. (M) Tumor growth curve of C57BL/6 mice subcutaneously inoculated with $1 \times 10^{6} \mathrm{RM} 1 \mathrm{cells}$. The mice were treated with antibodies to delete CD4+ T cells, CD8+ T cells and natural killer cells combined with USNB. The bars represent the mean $\pm S E M$; the $p$ value was calculated by unpaired t-test $(G-L)$. The data are representative of two independent experiments with at least six mice per group. dLN, draining lymph node; USNB, ultrasound-stimulated nanobubble.

is thought to be an important transcription factor of stem-like CD8+ T cells that can respond to PD1 blockade and enhance the antitumor response. ${ }^{34}$ The expression level of Tbet in CD44+CD8+ T cells was increased in the
USNB treatment group but not in the anti-PD1 treatment group, and USNB combined with anti-PD1 treatment further enhanced Tbet expression compared with USNB treatment (figure 3E). Moreover, we found that both 
A

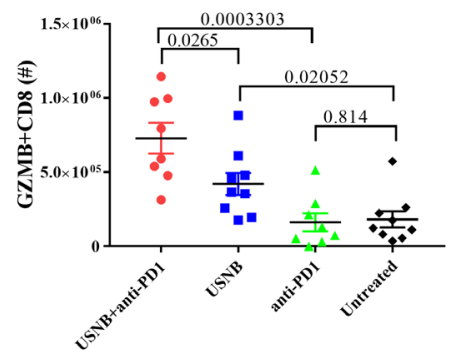

$\mathrm{D}$

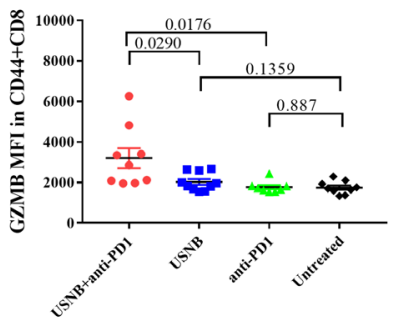

E

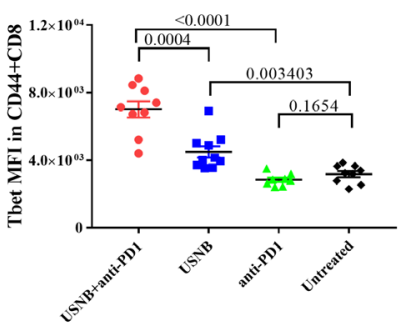

I

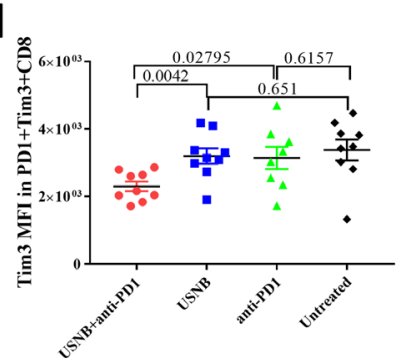

B

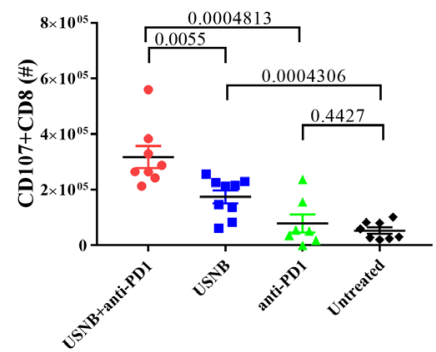

C

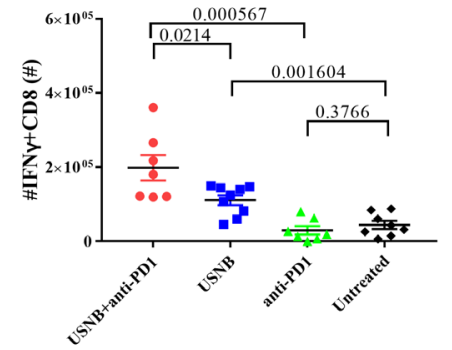

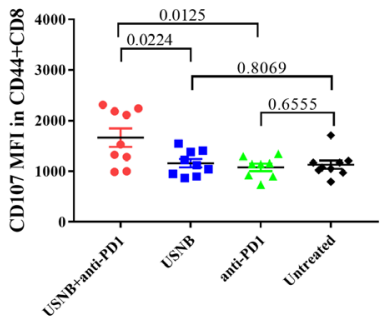
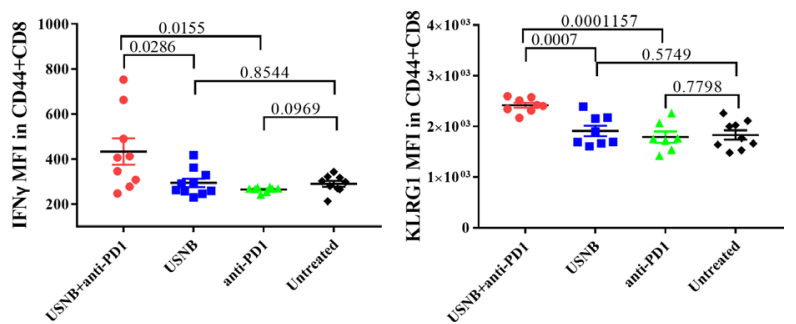

F

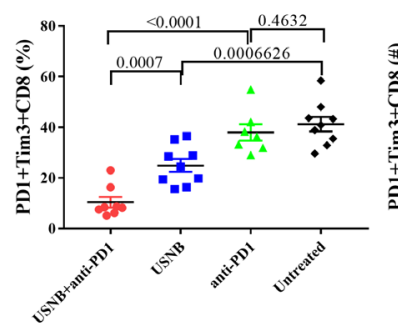

G
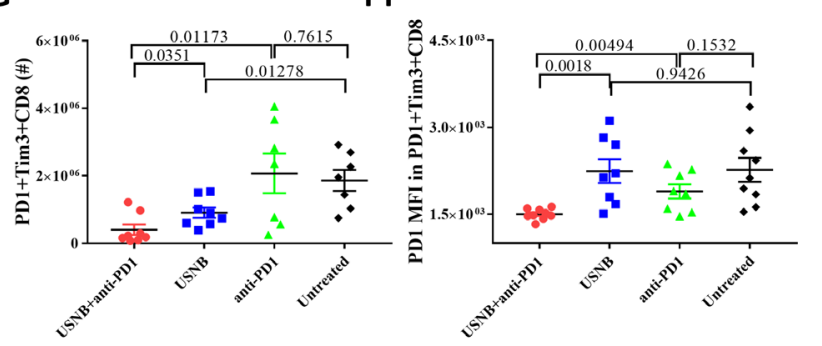

Figure 3 USNB treatment increases the antitumor effects and attenuates exhaustion of CD8+ T cells. C57BL/6 mice implanted with RM1 cells were left untreated or treated with anti-PD1, USNB or USNB+anti-PD1. The mice were sacrificed after the treatment schedule. The absolute number of GZMB+CD8+ T cells (A), CD107+CD8+ T cells (B) and IFN- $\gamma+C D 8+T$ cells (C) in tumor tissue. (D) MFI of GZMB, CD107, IFN- $\gamma$ and KLRG1 in CD44+CD8+ T cells. (E) MFI of Tbet in CD44+CD8+ T cells. $(F, G)$ Percentage and absolute number of PD1+Tim3+CD8+ T cells in tumor tissues, respectively. (H,I) MFI of PD1 and Tim3 in PD1+Tim3+CD8+ T cells, respectively. The bars represent the mean \pm SEM. The $p$ value was calculated by unpaired t-test $(A-I)$. The data are representative of two independent experiments with at least seven mice per group. IFN- $\gamma$, interferon gamma; $\mathrm{MFI}$, mean fluorescence intensity; USNB, ultrasound-stimulated nanobubble.

the percentage and number of PD1+Tim3+CD8+ T cells were decreased after USNB and combination treatment (figure 3F,G). In addition, the MFI of PD1 and Tim3 also decreased in the combination group (figure $3 \mathrm{H}, \mathrm{I}$ ), suggesting that the degree of CD8+ T-cell exhaustion was also decreased. These results indicate that combination treatment can significantly alleviate the exhaustion of CD8+ T cells. The corresponding data of the MC38 model are shown in online supplemental figure S3. Overall, these results demonstrate that USNB treatment upregulates the effect function of CD8+ T cells and enhances the expression of Tbet in CD8+ T cells, thereby improving the response to PD1 blockade, especially in immunologically cold tumors.

\section{USNB promotes the immune response by facilitating the release of tumor antigens and DAMPs}

The major mechanism that limits the effect function of $\mathrm{CD} 8+\mathrm{T}$ cells in cold tumors is their poor antigen exposure, and hence they are largely invisible to antigenpresenting cells (APCs). This limitation can be overcome by an inflammatory response, such as the immunogenic cell death of tumor cells, which can permit APCs to efficiently take up tumor antigens. ${ }^{14}$ To investigate whether 

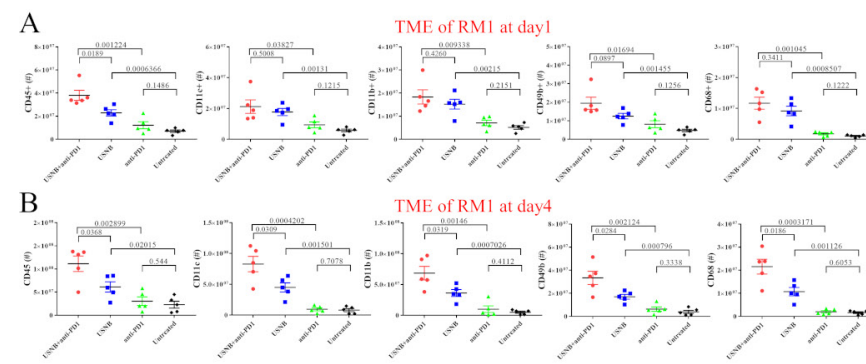

TME of RM1 at day 4
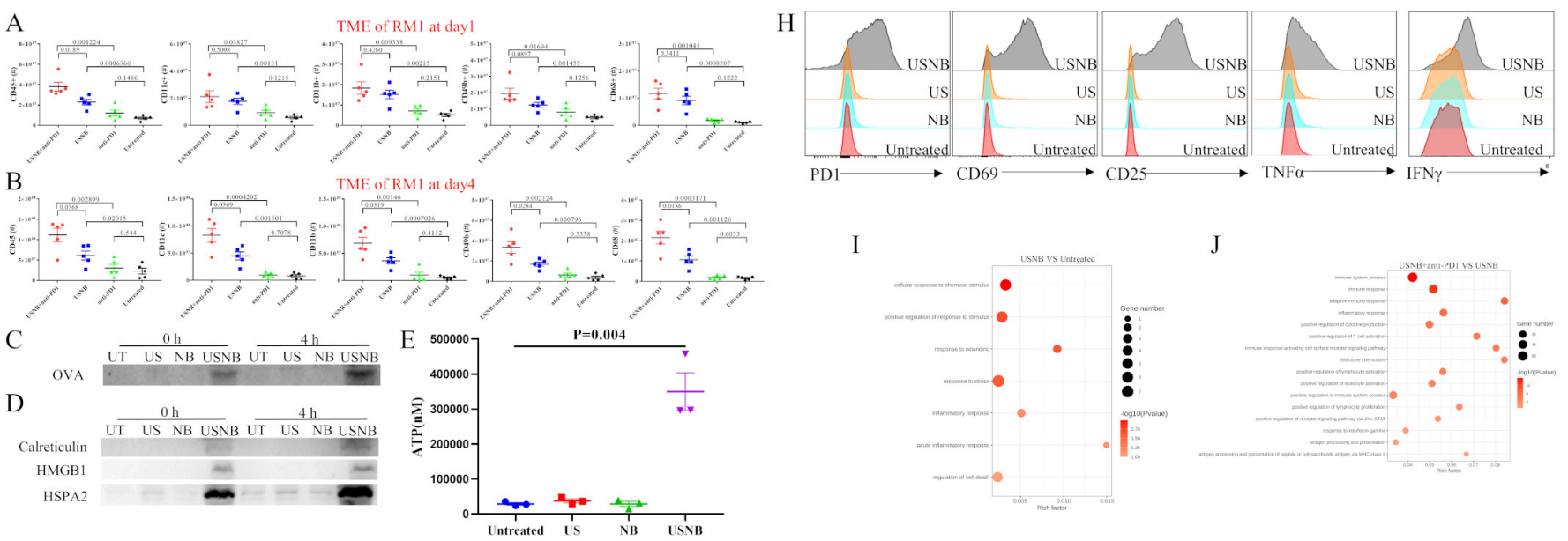

I

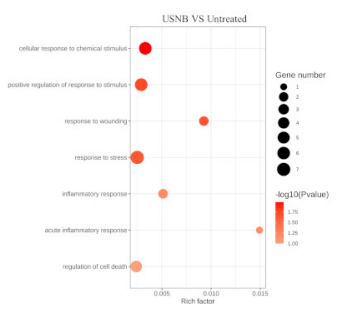

J
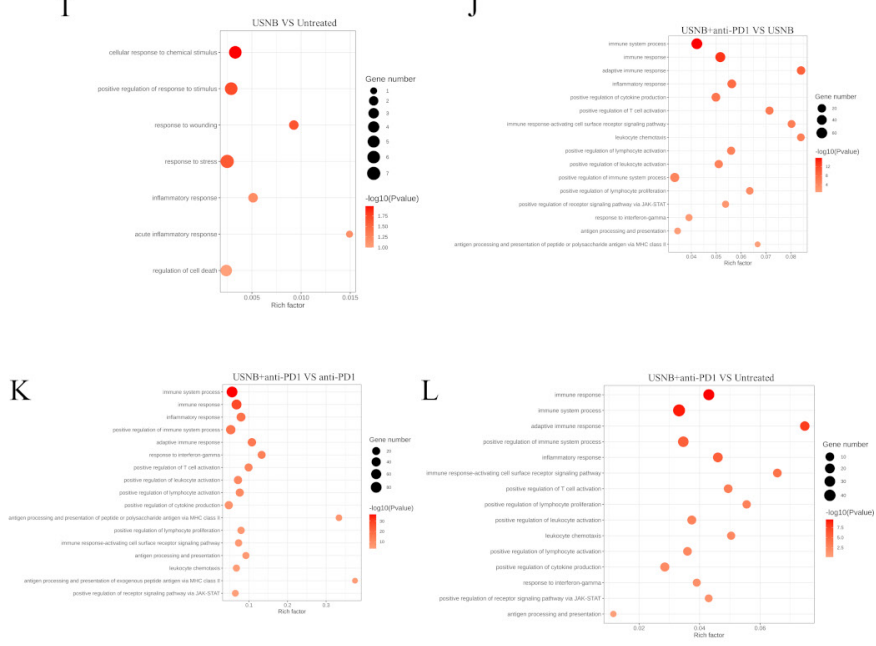

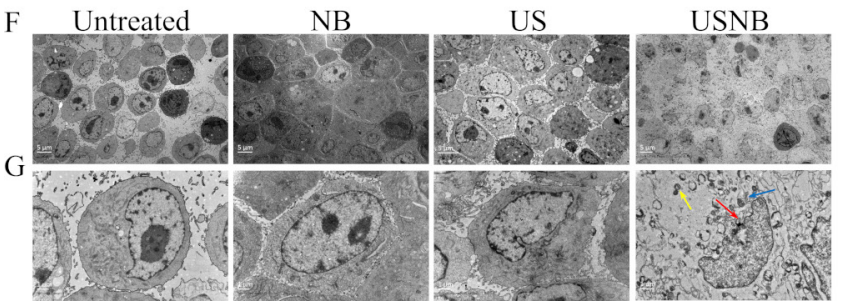

Figure 4 USNB lyses cells and causes immunogenic cell death. C57BL/6 mice implanted with RM1 cells were left untreated or were treated with anti-PD1, USNB or USNB+anti-PD1. One day (A) and 4 days (B) after treatment, the absolute numbers of leukocytes (CD45+), DCs (CD11c+), monocytes (CD11b+), NK cells (49b+) and macrophages (CD68+) in tumor tissue were analyzed by flow cytometry. RM1-OVA cells were treated with USNB, US or NB in vitro, and the supernatants were collected and analyzed. (C,D) Western blot of tumor antigen OVA levels and immunogenic cell death marker levels in the supernatants, respectively. (E) ATP concentration in the supernatants of RM1 cells in the different treatment groups. (F,G) Transmission electron microscopy images of RM1 cells obtained immediately after treatment with US, NB or USNB. The blue arrow indicates the fragmented Golgi apparatus; the yellow arrow indicates swollen mitochondria; and the red arrow indicates fragmented nuclei. The scale bars in the global and magnified images represent 5 and $1 \mu \mathrm{m}$, respectively. $(\mathrm{H})$ Flow cytometry analysis of PD1, CD69, CD25, TNF- $\alpha$ and IFN- $\gamma$ expression levels in CD8+ T cells after ex vivo coculture assay. Gene ontology analysis of differentially expressed genes in tumor masses between (l) USNB and untreated, (J) USNB+anti-PD1 and USNB, (K) USNB+anti-PD1 and anti-PD1, (L) USNB +anti-PD1 and untreated samples. The bars represent the mean \pm SEM $(n=3$ per group in $E)$. The $p$ value was calculated by unpaired t-test $(A, B, E)$. (A,B) The data are representative of two independent experiments with at least five mice per group. $(\mathrm{C}-\mathrm{H})$ The data are from three independent experiments, and representative results are shown. IFN- $\gamma$, interferon gamma; UT, untreated; NB, nanobubble; NK, natural killer; TME, tumor microenvironment; TNF- $\alpha$, tumor necrosis factor alpha; US, ultrasound; USNB, ultrasound-stimulated nanobubble.

the augmentation of the infiltration and effect function of CD8+ T cells is caused by APC-induced CD8+ T-cell activation, we further examined APCs, for example, CD11c+ (dendritic cells (DCs)) and CD68+ (macrophages) and other innate immune cells, such as CD11b+ (monocytes) and CD49b+ (NK cells), in the TME and dLNs of the RM1 tumor model at different time points (days 1 and 4) after treatment. In the TME, the numbers of leukocytes (CD45+), DCs, monocytes, NK cells and macrophages increased after USNB treatment alone or in combination with anti-PD1 treatment on both day 1 (figure 4A) and day 4 (figure 4B). In dLNs, the numbers of DCs, monocytes and macrophages were increased in the USNB and USNB+anti-PD1 groups compared with those in the untreated and anti-PD1 groups at day 1 (online supplemental figure $\mathrm{S} 4 \mathrm{~A}$ ), but this difference disappeared at day 4 (online supplemental figure S4B). The data of the MC38 tumor model are shown in online supplemental figure S4C-S4F. To further explore whether USNB can enhance tumor cell antigen exposure. We cultured RM1OVA cells in vitro and treated them with NB, US or USNB or left them untreated. Then, we examined OVA levels in the supernatant by western blot. USNB treatment notably increased the release of the OVA antigen into the supernatant (figure 4C). These results indicate that USNB can enhance antigen release and accumulation of innate immune cells, including APCs, in the TME and dLNs.

Another important approach that facilitates the antigen presentation function of APCs is inducing the release of DAMPs in tumor cells, ${ }^{35}$ so we examined the levels of DAMPs (HMGB1, ATP, calreticulin and HSPA2) in the supernatants of RM1-OVA cells after different treatments. The results showed that at both 0 and 4 hours after USNB treatment, HMGB1, calreticulin and HSPA2 levels were noticeably increased (figure 4D). ATP release was detected immediately after US, NB and USNB treatment, and the amount of ATP released by USNB-treated cells was obviously higher than that released by other cells 
(figure 4E). The morphology of cells after treatment was viewed by TEM. As shown in figure 4F,G, USNB-treated RM1 cells were severely damaged, as evidenced by the loss of cell membrane integrity, swollen mitochondria, fragmented nuclei and leakage of cell contents into the extracellular space. However, neither US nor NB treatment affected the morphology of RM1 cells. The same phenotype was observed in MC38 cells (online supplemental figure S5A-S5E). MitoTracker/7AAD staining of USNBtreated MC38 cells was then performed and evaluated under confocal microscopy. USNB-treated cells exhibited substantial changes in cell morphology and high levels of 7AAD+ nuclei fragments combined with some mitochondrial leakage from the cells (online supplemental figure $\mathrm{S} 5 \mathrm{~F})$; these factors are considered DAMPs that are able to initiate the immune response. Then, we cocultured splenocytes with the supernatants of NB-treated, US-treated and USNB-treated RM1-OVA cells. Flow cytometric staining analysis showed that $\mathrm{CD} 8+\mathrm{T}$ cells expressed higher levels of activation (PD1, CD69 and CD25) and killing (TNF- $\alpha$ and IFN- $\gamma$ ) markers (figure $4 \mathrm{H}$ ).

Consistently, the RNAseq data showed that after USNB treatment, the TME exhibited a high level of 'acute inflammation response' signaling pathway and other pathways related to 'cell death' and 'response to stimulation and wound' (figure 4I), which confirmed that USNB can cause tumor cell injury and promote inflammation and the immune response. Moreover, the USNB+anti-PD1 group also exhibited a high level of signaling pathways related to 'immune response', 'T-cell activation' and 'antigen processing and presentation' compared with the USNB, anti-PD1 or untreated groups (figure $4 \mathrm{~J}-\mathrm{L}$ ). Collectively, our findings strongly suggest that USNB induces antigen and DAMP release to enhance the therapeutic effect of PD1 blockade by improving antigen presentation in immunogenic cold tumors and further increasing the effector function of $\mathrm{CD} 8+\mathrm{T}$ cells.

\section{USNB causes necrosis of tumor cells to facilitate the release of tumor antigens and DAMPs}

As we have proven that USNB treatment alone can reduce tumor burden and lead to increased antigen and DAMP release via severe cellular injury, we hypothesized that USNB treatment can directly cause tumor cell necrosis. First, we confirmed that NB could be taken up by RM1 cells (figure 5A) and MC38 cells (online supplemental figure $\mathrm{S} 6 \mathrm{~A}$ ) as soon as $5 \mathrm{~min}$ after being added to the cell medium, and NB remained stable after internalization into the cytoplasm, suggesting that NB can enter tumor cells and remain intact before US stimulation.

To determine the effect of USNB on cells in vitro, we established four groups of RM1 cells: the untreated, US treatment, NB treatment and USNB treatment groups. As the results showed, the majority of cells broke into fragments after USNB treatment. After 24 hours, the cells in the US, NB and untreated groups had reattached to the flask and had grown to confluence, while the USNB-treated cells remained suspended and dying (figure 5B). Then, we evaluated the apoptosis of treated cells by staining for 7AAD, annexin $\mathrm{V}$ and caspase 3 at 0 and 4 hours after treatment. At 0 hour, approximately $65 \%$ of the USNBtreated cells were annexin $\mathrm{V}$ - and 7AAD+, while in the other treatment groups, the percentage of these cells was less than $1 \%$ (figure $5 \mathrm{C}$ ). Four hours after treatment, the annexin $\mathrm{V}-7 \mathrm{AAD}+$ population of USNB-treated cells was reduced to $7.84 \%$, and the annexin $\mathrm{V}+7 \mathrm{AAD}+$ population was increased to approximately $21.8 \%$ (figure $5 \mathrm{D}$ ). At 0 hour, caspase 3 expression was comparable among these groups (figure 5E), but 4 hours after treatment, caspase 3 expression was increased by $43.3 \%$ in the USNB group compared with that in other groups (figure $5 \mathrm{~F}$ ). These results indicate that USNB leads to immediate cell death via the cavitation effect and can later increase apoptotic signals in surviving cells. Then, we used CFSE-labeled RM1 cells to evaluate the growth of tumor cells 24 hours after USNB treatment. The USNB-treated cells did not grow, whereas other treatments had no apparent impact on cell growth (figure 5G). Moreover, the MC38 data are shown in online supplemental figure S6B-S6G, and the data are consistent with those of the RM1 tumor model. In addition, ROS production in MC38 cells was obviously increased 4 hours after USNB treatment (online supplemental figure $\mathrm{S} 6 \mathrm{H}$ ). Overall, these results confirmed that the mechanical force provided by USNB-induced cavitation activity can directly lead to immediate cell necrosis in vitro.

We further investigated this bioeffect of USNB in vivo. A larger necrotic region was produced under USNB treatment conditions, while the US and untreated groups exhibited little necrosis in both the RM1 (figure 5H,I) and MC38 (online supplemental figure S6I,J) tumor models. These results confirmed that USNB can lead to tumor necrosis both in vivo and in vitro, while US treatment alone could not, indicating that the effect of USNB on cells does not rely on the thermal effect of US but relies on the mechanical force of NB stimulated by US.

\section{USNB treatment in combination with PD1 blockade enhances systemic antitumor immunity and immune memory}

Metastatic lesions are the main obstacle in clinical cancer therapy, and the activation of systemic antitumor immune response can effectively inhibit metastases. We inoculated RM1 tumors on both legs of mice and treated the mice with USNB, anti-PD1 or USNB+anti-PD1, with USNB applied to the tumor in the right leg (figure 6A). As expected, the growth pattern of the USNB-treated tumors on the right leg was consistent with previous data (figure 6B). Impressively, the growth curve of the untreated tumors exhibited the same trend (figure 6C). Next, we repeated the same experiment in the MC38 tumor model and obtained the same results (figure 6D-F). In addition, we injected $0.3 \times 10^{6}$ MC38 tumor cells intravenously 3 days after the subcutaneous implantation of $0.7 \times 10^{6}$ MC38 cells into the right leg, and USNB was applied to the subcutaneous tumor (figure 6G). The growth pattern of the USNB-treated tumors was consistent with previous 

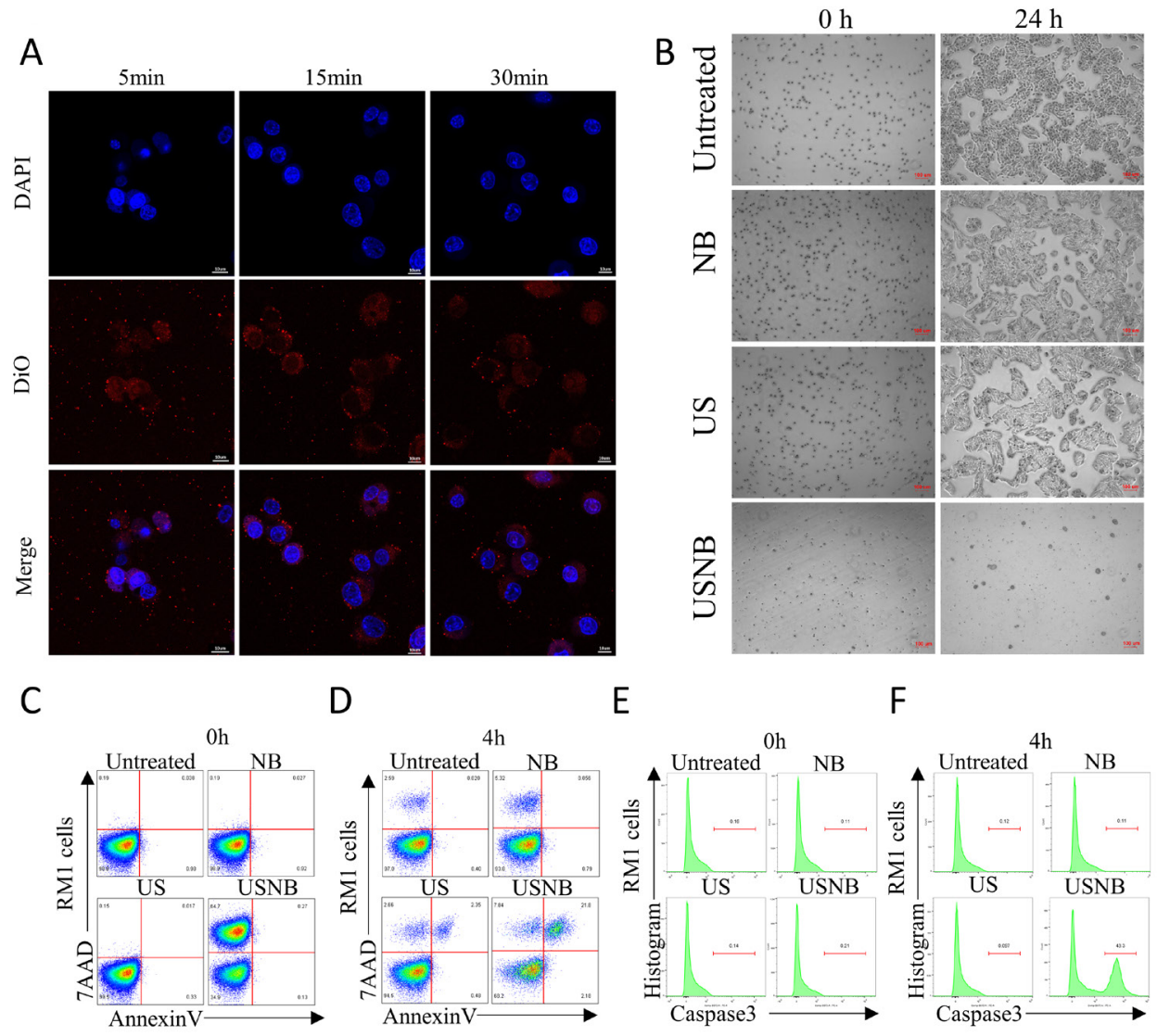

E

F
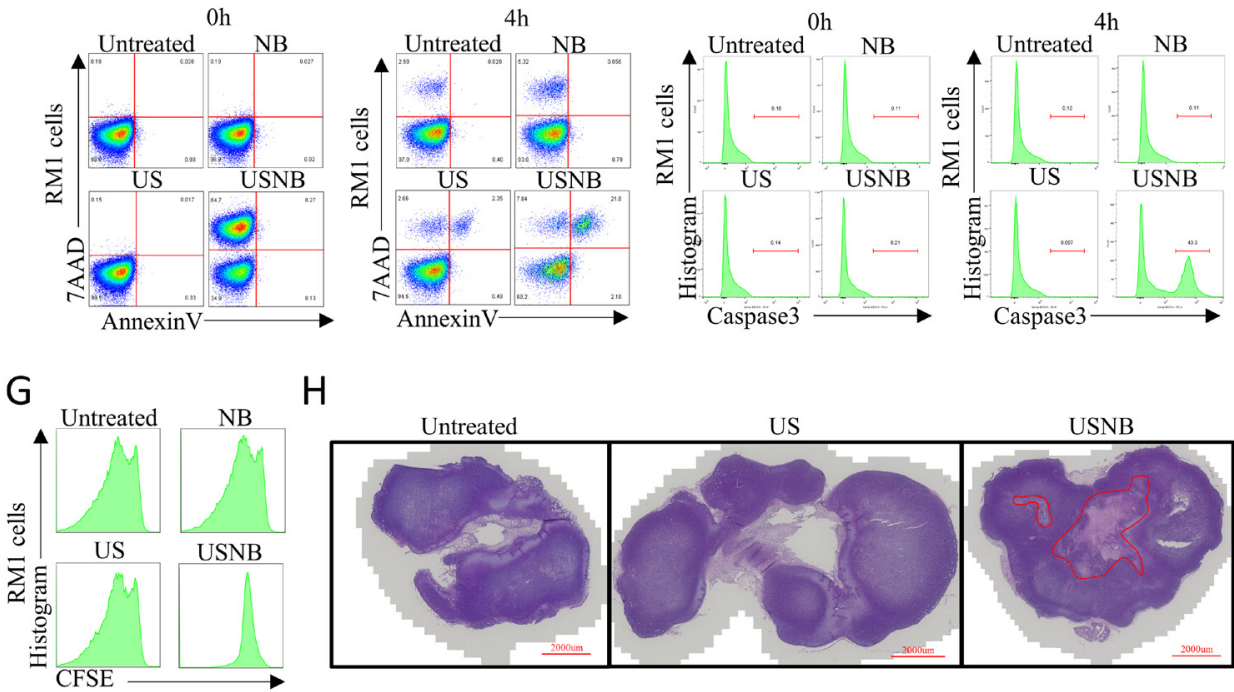

$\mathrm{H}$
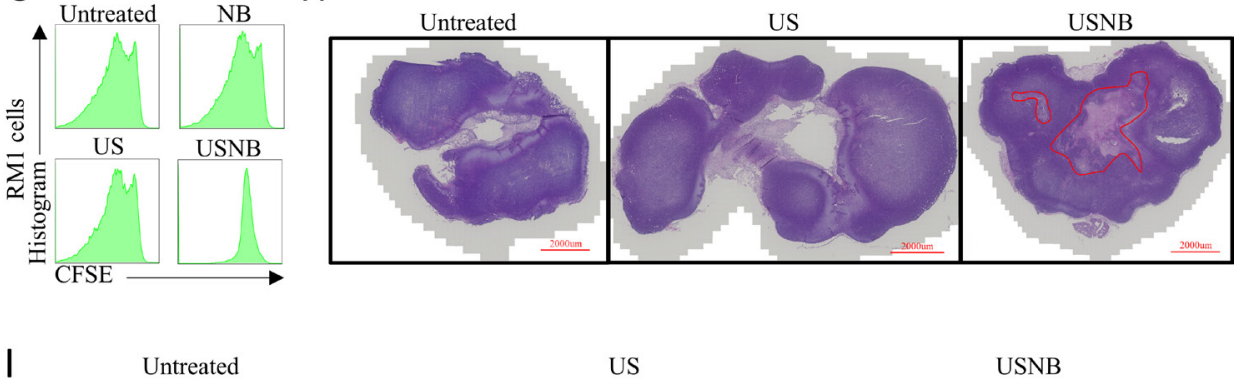

US

USNB

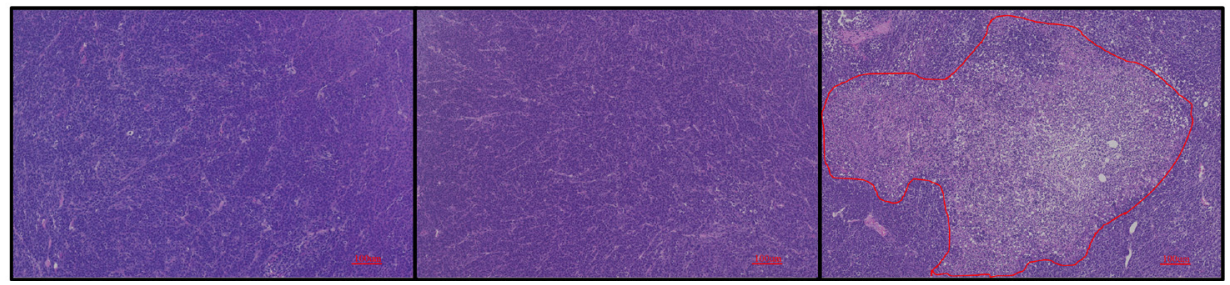

Figure 5 Effect of USNB on tumor cells and tissues. (A) Confocal microscopy images of RM1 cells after NB treatment for 5 , 15 and 30 min. Nuclei were stained with DAPI, and NBs were stained with DiO. The scale bar in the image represents $10 \mu \mathrm{m}$. (B) USNB were used to treat RM1 cells in vitro, and the morphology was observed by microscopy at 0 hour (left) and 24 hours (right) after treatment. The scale bars in the images represent $100 \mu \mathrm{m}$. At 0 and 4 hours after the indicated treatments, RM1 cells were stained with 7AAD and annexin V (C,D) or caspase3 antibodies (E,F) and analyzed by flow cytometry. (G) Histogram of CFSE staining, indicating the proliferation ability of RM1 cells 24 hours after treatment. $(\mathrm{H}, \mathrm{I}) \mathrm{H} \& \mathrm{E}$ staining of tumor tissues after the mice were sacrificed. the red zone indicates the necrosis region. The scale bars in the global and magnified images represent 2000 and $100 \mu \mathrm{m}$, respectively. (A-G) The data are from three independent experiments, and representative results are shown. $(\mathrm{H}, \mathrm{I})$ The data are representative of two independent experiments with at least five mice per group. DAPI, 4',6-diamidino2-phenylidole; NB, nanobubble; US, ultrasound; USNB, ultrasound-stimulated nanobubble.

data (figure $6 \mathrm{H}$ ). The number of metastatic nodes was significantly lower in the USNB+anti-PD1 group than in the untreated and anti-PD1 antibody-treated groups.
Notably, anti-PD1 treatment did not significantly control the formation of metastatic foci (figure 6I and online supplemental figure S7). Collectively, these results proved 
A

$1 \mathrm{M}$<smiles>CCCCC1COCC2CCC(CC2)C1CC(C)(C)C</smiles>

D

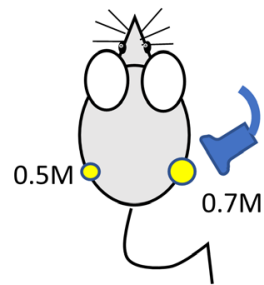

G

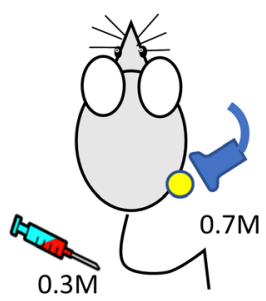

J
B

E

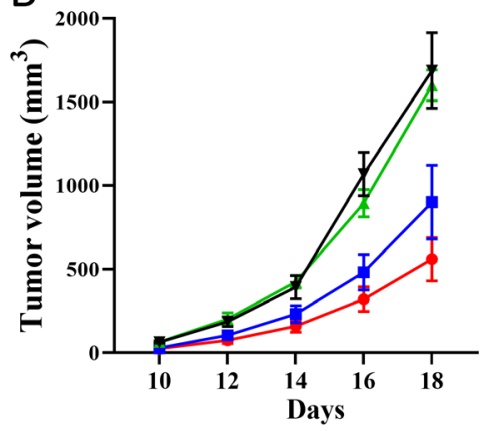

C

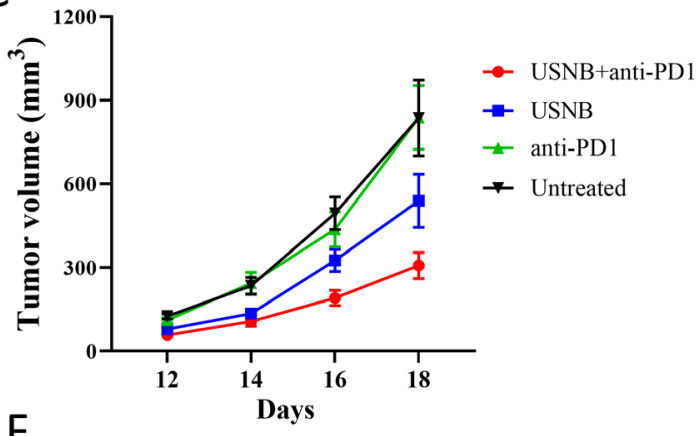

$\mathrm{H}$
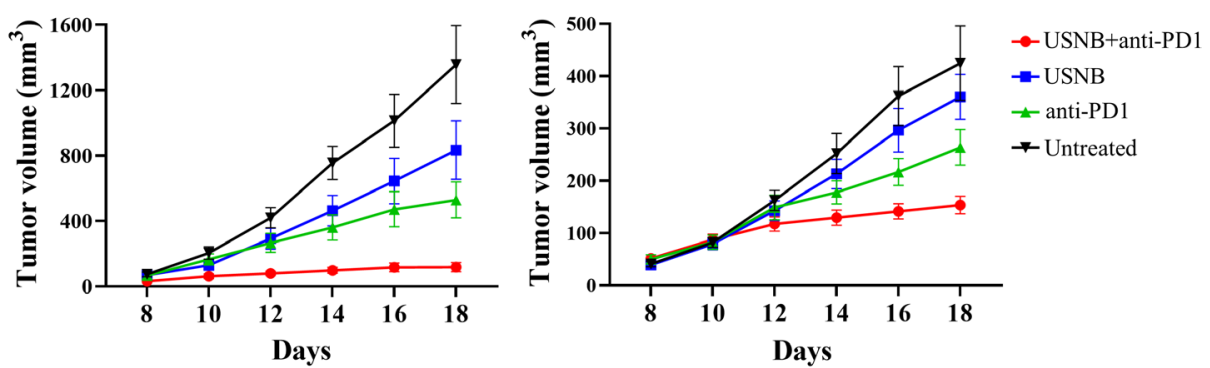

I

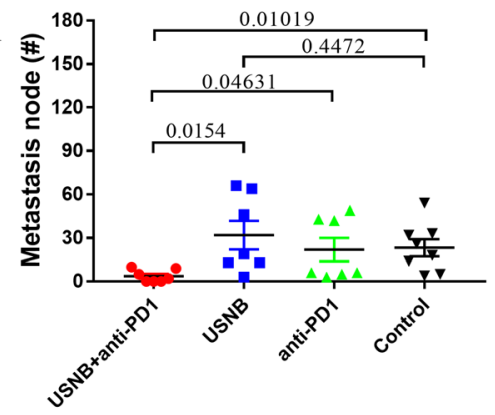

Rechallenge

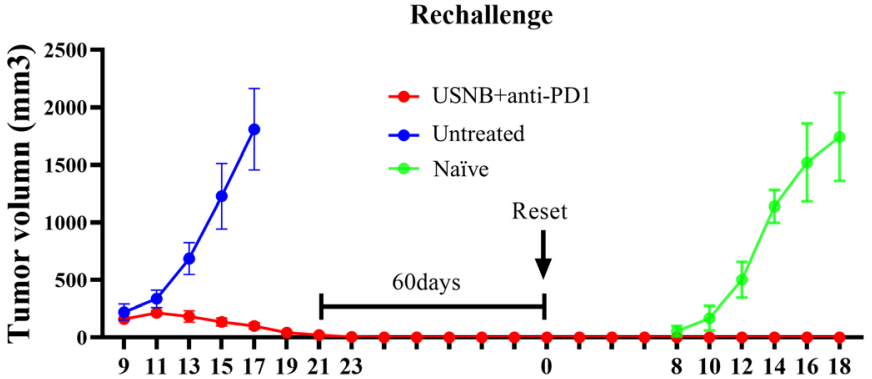

Days

Figure 6 USNB combined with PD1 blockade treatment exerts abscopal effects and promotes immune memory.

(A) Schematic diagram of the experimental design. Briefly, RM1 cells were subcutaneously inoculated into C57BL/6 mice, with $1.2 \times 10^{6}$ cells inoculated on the right side and $1 \times 10^{6}$ cells inoculated on the opposite side 3 days later. The mice were divided into the untreated, USNB-treated, anti-PD1-treated and combination-treated groups. USNB treatment was applied on the right side when the tumor size reached $50 \mathrm{~mm}^{3}$. (B,C) The tumor growth curves of the USNB-treated (right) side and untreated (opposite) side, respectively. (D) Schematic diagram of the study design. Briefly, MC38 cells were subcutaneously inoculated into C57BL/6 mice, with $0.7 \times 10^{6}$ cells inoculated on the right side and $0.5 \times 10^{6}$ cells inoculated on the opposite side 3 days later. USNB treatment was applied on the right side when the tumor size reached $50 \mathrm{~mm}^{3}$. (E,F) The tumor growth curves of the USNB-treated (right) side and untreated (opposite) side, respectively. (G) Schematic diagram of the study design. Briefly, $0.7 \times 10^{6} \mathrm{MC} 38$ cells were subcutaneously inoculated into C57BL/ 6 mice, and 3 days later, $0.3 \times 10^{6}$ cells were injected intravenously. USNB treatment was applied subcutaneously after the tumor reached $50 \mathrm{~mm}^{3}$. (H) Tumor growth curve of USNBtreated subcutaneous tumors. (I) Number of tumor nodules in the lungs in each group. (J) Growth curve of mice with CR treated with the USNB and PD1 blockade combination and rechallenged with $0.5 \times 10^{6} \mathrm{MC} 38$ cells. The bars represent the mean \pm SEM. The $p$ value was calculated by unpaired t-test $(F)$. The data are representative of two independent experiments with at least seven mice per group. USNB, ultrasound-stimulated nanobubble. 
that USNB treatment exerts an abscopal effect and can effectively promote systemic antitumor immunity, leading to elimination of distal untreated tumors, which is crucial for advanced cancer patients in the clinic. Then, we conducted a rechallenge assay 60 days after complete remission (CR) of the MC38 tumor masses and found that mice with $\mathrm{CR}$ acquired immune memory protection and completely eliminated the MC38 tumor cells (figure 6J). These results indicated that USNB treatment in combination with PD1 blockade enhanced systemic antitumor immunity and promoted the acquisition of immune memory after complete remission.

\section{CONCLUSION}

In this study, we reveal that the combination of USNB and anti-PD1 therapy effectively inhibits the growth and recurrence of cold tumors, providing evidence that US-targeted NBs can serve as a novel immunological adjuvant strategy for immunotherapy. Importantly, we demonstrated for the first time that USNB reprograms cold tumors into hot tumors by stimulating tumor antigen and DAMPs release, thus sustaining systemic antitumor immunity and acquired immune memory after complete remission.

Recently, the induction of antitumor effects by lowintensity US-stimulated sonosensitizers carried by NBs (eg, Ce6 and ICG carried by NBs) has emerged as an approach for enhancing cancer immunotherapy, ${ }^{36} 37$ but it is still difficult to apply sonosensitizer-labeled NBs in clinical practice. In this study, USNB treatment alone was found to exert antitumor effects comparable to those of sonosensitizer-labeled NBs, and our NBs can be easily prepared; therefore, they are more practical for clinical use. In addition, US can lead to tumor fixation, which blocks antigen release and immune cell penetration, and consequently causes a reduced immune response. ${ }^{38} 39$ Therefore, we applied low-intensity and low thermal exposure of US to debulk tumor cells through cavitationinduced mechanical fragment production and to facilitate the release of DAMPs to stimulate the immune response and improve immunotherapy efficacy; this approach can decrease US-induced heat convection-associated iatrogenic effects and will not affect the blood supply to the tumor or antitumor immune cell infiltration. This is a revolution in current clinical tumor treatment strategies and provides a new option for combination therapy for patients suffering from immunotherapy-resistant tumors.

In our study, we demonstrate three potential mechanisms of action for this treatment. USNB treatment led to accidental disintegration of tumor cells through the acoustic cavitation effect. Tumor cells exhibit significantly high levels of 7AAD and ROS after USNB treatment. Immediate cell lysis causes the release of cell contents, such as damaged mitochondria, endoplasmic reticulum and antigens, into the TME. In addition, cell disruption caused by USNB releases DAMPs, such as HSPA2, HMGB1, calreticulin and DNA fragments. Damaged mitochondria could lead to the release of large amounts of ROS and DAMPs, such as mtDNA and other mitochondrial proteins. ${ }^{40-42}$ DAMPs are endogenous immunogenic molecules that can stimulate pattern recognition receptors, such as toll-like receptors, nucleotide-binding domains and RIG-I-like receptors, thereby promoting an inflammatory response and an antitumor immune response by activating APCs. ${ }^{43}$ It has been reported that DAMPs function as an 'eat me' signal for DCs and are ingested via macropinocytosis. ${ }^{44}$ This phenomenon occurs only on the loss of cell membrane integrity; therefore, tumor cell necrosis after USNB treatment, but not tumor cell apoptosis after chemotherapy or irradiation therapy, can achieve this effect. These factors suggest that the sudden mechanical damage caused by USNB leads to tumor necrosis and DAMP release, which creates a TME that is optimal for immune cell infiltration and antigen presentation. We also observed the enrichment of immune cell infiltration, including the infiltration of leukocytes, NK cells and APCs, such as DCs and macrophages, in the dLNs and TME after USNB treatment. The significant accumulation of APCs in dLNs and the TME indicates better priming and stimulation of $\mathrm{T}$ lymphocytes. ${ }^{45}$ Our results also showed that the number of CD44+CD8+ T cells was increased in both tumors and dLNs, indicating an increase in tumor-specific CD8+ $\mathrm{T}$ cells ready to mount a positive response to immunotherapy. Moreover, the expression level of Tbet, a marker of stem-like CD8+ T cells, the main subset of the anti-PD1 response, was also increased in USNB-treated mice. This evidence demonstrated that combining USNB and antiPD1 enhanced antitumor immunity.

Importantly, we found that when USNB was combined with PD1 blockade, the number and effector function of CD8+ T cells were systemically increased, which were accompanied by a reduced state of exhaustion, leading to improved systemic antitumor immunity and abscopal effects. This effect strongly suggests a promising role of USNB treatment even in those tumor lesions where US cannot reach or detect, like lung metastases. The same phenomenon was observed in mouse prostate cancer and colon cancer xenograft models, suggesting that USNB treatment can be widely applied to solid tumors to enhance systemic antitumor immunity. Long-term immune memory protection occurred after complete MC38 remission, and $100 \%$ of mice with CR successfully neutralized an MC38 rechallenge. We therefore envision this strategy in a setting with early-stage cancer. In this scenario, USNB combined with PD1 blockade could be used to eliminate tumor cells and induce antitumor immunity, which then provide long-term antitumor memory protection.

Despite potent antitumor efficacy and synergistic effects with anti-PD1 therapy, this combination therapy has some limitations. Although NB can pass through blood vessels and accumulate in tumor tissue via passive targeting, a large number of NB remain in the peripheral blood. Thus, new strategies for guiding NB into the TME and to sites closely adjacent to tumor cells should be further 
explored in the future. A recent study showed that NB modification with a cell-penetrating peptide $\left(\mathrm{HE}_{6} \mathrm{R}_{6}\right)$ can facilitate membrane binding and cellular uptake, therefore promoting the internalization of $\mathrm{NBs}^{46}$ which provides us with a reference for optimizing our NB and combination therapy in mouse trials and clinical trials.

In summary, our results demonstrate that USNB treatment can efficiently reprogram cold tumors into hot tumors to benefit from immunotherapy. In addition, the combination of USNB and anti-PD1 therapy can achieve long-lasting inhibition of tumor growth, metastasis and recurrence by inducing a systematic immune response and long-term memory protection. Our study thus presents USNB treatment as a promising strategy for sensitizing cold tumors to immunotherapy in the clinic.

Acknowledgements We thank Professor Lilin Ye (Army Medical University) and Professor Jianxiang Zhang (Army Medical University) for generously providing guidance and technical assistance for our project. We thank Jiayin Biotechnology (Shanghai, China) for assistance with the RNA-seq assay.

Contributors $\mathrm{JHu}$, JHe and $\mathrm{YW}$ designed and supervised the study with $\mathrm{YG}, \mathrm{HL}$ and J0; YD, YC, YZhan, CZ, HW, JT, JW, RZ and CL performed the experiments; YZhao and KF analyzed the data; and JHu and JHe wrote the manuscript. All the authors approved the content of the final version of the manuscript. J0: guarantor.

Funding This work was supported in part by grant numbers 82072745 (J0), 81772647 (JO), 81874072 (HL) and 81771856 (YG) from the National Natural Science Foundation of China.

Competing interests None declared.

Patient consent for publication Not applicable.

Ethics approval This study does not involve human subjects. All the mouse experiments were conducted in accordance with the guidelines of the Laboratory Animal Welfare and Ethics Committee of Third Military Medical University (approval number AMUWEC20191575).

Provenance and peer review Not commissioned; externally peer reviewed.

Data availability statement All data relevant to the study are included in the article or uploaded as supplementary information. All data supporting the findings of this study are available within the article and its online supplemental information files and from the corresponding author on reasonable request.

Supplemental material This content has been supplied by the author(s). It has not been vetted by BMJ Publishing Group Limited (BMJ) and may not have been peer-reviewed. Any opinions or recommendations discussed are solely those of the author(s) and are not endorsed by BMJ. BMJ disclaims all liability and responsibility arising from any reliance placed on the content. Where the content includes any translated material, BMJ does not warrant the accuracy and reliability of the translations (including but not limited to local regulations, clinical guidelines, terminology, drug names and drug dosages), and is not responsible for any error and/or omissions arising from translation and adaptation or otherwise.

Open access This is an open access article distributed in accordance with the Creative Commons Attribution Non Commercial (CC BY-NC 4.0) license, which permits others to distribute, remix, adapt, build upon this work non-commercially, and license their derivative works on different terms, provided the original work is properly cited, appropriate credit is given, any changes made indicated, and the use is non-commercial. See http://creativecommons.org/licenses/by-nc/4.0/.

\section{ORCID iD}

Jianjun Hu http://orcid.org/0000-0001-5393-880X

\section{REFERENCES}

1 Jin Q, Zhu W, Zhu J, et al. Nanoparticle-Mediated delivery of inhaled immunotherapeutics for treating lung metastasis. Adv Mater 2021;33:e2007557:2007557.

2 Matzner P, Sandbank E, Neeman E, et al. Harnessing cancer immunotherapy during the unexploited immediate perioperative period. Nat Rev Clin Oncol 2020;17:313-26.
3 Ganesh K, Stadler ZK, Cercek A, et al. Immunotherapy in colorectal cancer: rationale, challenges and potential. Nat Rev Gastroenterol Hepatol 2019;16:361-75.

4 Phuengkham H, Song C, Lim YT. A designer scaffold with immune Nanoconverters for reverting immunosuppression and enhancing immune checkpoint blockade therapy. Adv Mater 2019;31:e1903242:1903242.

5 Hashimoto M, Kamphorst AO, Im SJ, et al. Cd8 T cell exhaustion in chronic infection and cancer: opportunities for interventions. Annu Rev Med 2018;69:301-18.

6 Li J, Lee Y, Li Y, et al. Co-inhibitory Molecule B7 Superfamily Member 1 Expressed by Tumor-Infiltrating Myeloid Cells Induces Dysfunction of Anti-tumor CD8 ${ }^{+}$T Cells. Immunity 2018;48:773-86.

7 Zeng D-Q, Yu Y-F, Ou Q-Y, et al. Prognostic and predictive value of tumor-infiltrating lymphocytes for clinical therapeutic research in patients with non-small cell lung cancer. Oncotarget 2016;7:13765-81.

8 Liu D, Jenkins RW, Sullivan RJ. Mechanisms of resistance to immune checkpoint blockade. Am J Clin Dermatol 2019;20:41-54.

9 Chen P-L, Roh W, Reuben A, et al. Analysis of immune signatures in longitudinal tumor samples yields insight into biomarkers of response and mechanisms of resistance to immune checkpoint blockade. Cancer Discov 2016;6:827-37.

10 Tumeh PC, Harview CL, Yearley JH, et al. PD-1 blockade induces responses by inhibiting adaptive immune resistance. Nature 2014;515:568-71.

11 Galon J, Costes A, Sanchez-Cabo F, et al. Type, density, and location of immune cells within human colorectal tumors predict clinical outcome. Science 2006;313:1960-4.

12 Mlecnik B, Tosolini M, Kirilovsky A, et al. Histopathologic-based prognostic factors of colorectal cancers are associated with the state of the local immune reaction. J Clin Oncol 2011;29:610-8.

13 Ros XR, Vermeulen L. Turning cold tumors hot by blocking TGF- $\beta$. Trends Cancer 2018;4:335-7.

14 Sharma P, Allison JP. The future of immune checkpoint therapy. Science 2015;348:56-61.

15 Liu J, Yan J, Yan S, et al. Biomimetic and self-assembled nanoclusters targeting $\beta$-catenin for potent anticancer therapy and enhanced immunotherapy. Nano Lett 2019;19:8708-15.

16 Yamamoto K, Venida A, Yano J, et al. Autophagy promotes immune evasion of pancreatic cancer by degrading MHC-I. Nature 2020;581:100-5.

17 Spranger S, Bao R, Gajewski TF. Melanoma-intrinsic $\beta$-catenin signalling prevents anti-tumour immunity. Nature 2015;523:231-5.

18 Isazadeh A, Hajazimian S, Garshasbi H, et al. Resistance mechanisms to immune checkpoints blockade by monoclonal antibody drugs in cancer immunotherapy: Focus on myeloma. J Cell Physiol 2021;236:791-805

19 Galluzzi L, Senovilla L, Zitvogel L, et al. The secret ally: immunostimulation by anticancer drugs. Nat Rev Drug Discov 2012;11:215-33.

20 Smyth MJ, Ngiow SF, Ribas A, et al. Combination cancer immunotherapies tailored to the tumour microenvironment. Nat Rev Clin Oncol 2016;13:143-58.

21 Um W, Ko H, You DG, et al. Necroptosis-inducible polymeric nanobubbles for enhanced cancer sonoimmunotherapy. Adv Mater 2020;32:e1907953:1907953.

22 Erwig L-P, Henson PM. Clearance of apoptotic cells by phagocytes. Cell Death Differ 2008;15:243-50.

23 Tesniere A, Panaretakis T, Kepp O, et al. Molecular characteristics of immunogenic cancer cell death. Cell Death Differ 2008;15:3-12.

24 Deshpande PP, Biswas S, Torchilin VP. Current trends in the use of liposomes for tumor targeting. Nanomedicine 2013;8:1509-28.

25 Li N, Tang J, Yang J, et al. Tumor perfusion enhancement by ultrasound stimulated microbubbles potentiates PD-L1 blockade of MC38 colon cancer in mice. Cancer Lett 2021;498:121-9.

26 Bulner S, Prodeus A, Gariepy J, et al. Enhancing checkpoint inhibitor therapy with ultrasound stimulated microbubbles. Ultrasound Med Biol 2019;45:500-12.

27 Hobbs SK, Monsky WL, Yuan F, et al. Regulation of transport pathways in tumor vessels: role of tumor type and microenvironment. Proc Natl Acad Sci U S A 1998;95:4607-12.

28 Hashizume H, Baluk P, Morikawa S, et al. Openings between defective endothelial cells explain tumor vessel leakiness. $A m \mathrm{~J}$ Pathol 2000;156:1363-80.

29 Sano K, Nakajima T, Choyke PL, et al. The effect of photoimmunotherapy followed by liposomal daunorubicin in a mixed tumor model: a demonstration of the super-enhanced permeability and retention effect after photoimmunotherapy. Mol Cancer Ther 2014;13:426-32. 
30 Shen D, Zhu L, Liu Y, et al. Efficacy evaluation and mechanism study on inhibition of breast cancer cell growth by multimodal targeted nanobubbles carrying AMD070 and ICG. Nanotechnology 2020;31:245102.

31 Peng Y, Zhu L, Wang L, et al. Preparation of nanobubbles modified with a small-molecule CXCR4 antagonist for targeted drug delivery to tumors and enhanced ultrasound molecular imaging. Int $\mathrm{J}$ Nanomedicine 2019;14:9139-57.

32 Zhao M, Yang X, Fu H, et al. Immune/hypoxic tumor microenvironment regulation-enhanced photodynamic treatment realized by $\mathrm{pH}$-responsive phase transition-targeting nanobubbles. ACS Appl Mater Interfaces 2021;13:32763-79.

33 Cerwenka A, Lanier LL. Natural killers join the fight against cancer. Science 2018;359:1460-1.

34 Jansen CS, Prokhnevska N, Master VA, et al. An intra-tumora niche maintains and differentiates stem-like CD8 T cells. Nature 2019;576:465-70.

35 Lin S-Y, Hsieh S-Y, Fan Y-T, et al. Necroptosis promotes autophagydependent upregulation of dAMP and results in immunosurveillance. Autophagy 2018;14:778-95.

$36 \mathrm{Xu} \mathrm{J}, \mathrm{Xu} \mathrm{L}$, Wang C, et al. Near-infrared-triggered photodynamic therapy with multitasking upconversion nanoparticles in combination with checkpoint blockade for immunotherapy of colorectal cancer. ACS Nano 2017;11:4463-74.

37 Chen Q, Xu L, Liang C, et al. Photothermal therapy with immuneadjuvant nanoparticles together with checkpoint blockade for effective cancer immunotherapy. Nat Commun 2016;7:13193.
38 Eranki A, Srinivasan P, Ries M, et al. High-intensity focused ultrasound (HIFU) triggers immune sensitization of refractory murine neuroblastoma to checkpoint inhibitor therapy. Clin Cancer Res 2020;26:1152-61.

39 Silvestrini MT, Ingham ES, Mahakian LM, et al. Priming is key to effective incorporation of image-guided thermal ablation into immunotherapy protocols. JCl Insight 2017;2:e90521.

40 Kazama H, Ricci J-E, Herndon JM, et al. Induction of immunological tolerance by apoptotic cells requires caspase-dependent oxidation of high-mobility group box-1 protein. Immunity 2008;29:21-32.

41 Garg AD, Nowis D, Golab J, et al. Immunogenic cell death, DAMPs and anticancer therapeutics: an emerging amalgamation. Biochim Biophys Acta 2010;1805:53-71.

42 Duprez L, Takahashi N, Van Hauwermeiren F, et al. Rip kinasedependent necrosis drives lethal systemic inflammatory response syndrome. Immunity 2011;35:908-18.

43 Krysko DV, Garg AD, Kaczmarek A, et al. Immunogenic cell death and DAMPs in cancer therapy. Nat Rev Cancer 2012;12:860-75.

44 Krysko DV, Denecker G, Festjens N, et al. Macrophages use different internalization mechanisms to clear apoptotic and necrotic cells. Cell Death Differ 2006;13:2011-22.

45 Turley SJ, Fletcher AL, Elpek KG. The stromal and haematopoietic antigen-presenting cells that reside in secondary lymphoid organs. Nat Rev Immunol 2010;10:813-25.

$46 \mathrm{Yu}$ Y, Zu C, He D, et al. pH-Dependent reversibly activatable cellpenetrating peptides improve the antitumor effect of artemisininloaded liposomes. J Colloid Interface Sci 2021;586:391-403. 
Correction: Ultrasound combined with nanobubbles promotes

systemic anticancer immunity and augments anti-

PD1 efficacy

$\mathrm{Hu} \mathrm{J}$, He J, Wang Y, et al. Ultrasound combined with nanobubbles promotes systemic anticancer immunity and augments anti-PD1 efficacy. J Immunother Cancer 2022; 10: e003408.

This article has been corrected since it was first published to add Professor Houjie Liang and Professor Yanli Guo as corresponding authors.

Open access This is an open access article distributed in accordance with the Creative Commons Attribution Non Commercial (CC BY-NC 4.0) license, which permits others to distribute, remix, adapt, build upon this work non-commercially, and license their derivative works on different terms, provided the original work is properly cited, appropriate credit is given, any changes made indicated, and the use is non-commercial. See http://creativecommons.org/licenses/by-nc/4.0/.

C Author(s) (or their employer(s)) 2022. Re-use permitted under CC BY-NC. No commercial re-use. See rights and permissions. Published by BMJ.

J Immunother Cancer 2022;10:e003408corr1. doi:10.1136/jitc-2021-003408corr1

D) Check for updates 